\title{
Asteroid Bodies in Lymph Node Cytology: Infrequently Seen and Still Mysterious
}

\author{
Julie M. Jorns, M.D. and Stewart M. Knoepp, M.D., Ph.D.
}

Granulomatous inflammation is a relatively common finding in routine aspiration cytology of lymph nodes. However, asteroid bodies are very rarely encountered in cytologic preparations, and most morphologic descriptions result from observations made in histologic tissue sections. This brief report describes the cytologic findings in paratracheal aspirate smears from a 74-year-old Caucasian woman with the history of squamous-cell carcinoma of the right ankle metastasic to a right groin lymph node. At the time of removal of the metastatic tumor, the patient was noted to have multiple small, mildly FDG-avid lymph nodes in the supraclavicular, paratracheal, precarinal, pulmonic hilar, and axillary regions. A transbronchial fine-needle aspiration biopsy $(F N A B)$ of a paratracheal lymph node showed granulomatous inflammation and numerous multinucleated giant cells containing asteroid bodies. No evidence of malignancy was present in any of the smears. Additional patient history elicited at the time of FNAB revealed a diagnosis 6 years previously of disseminated histoplasmosis infection. A concomitant workup for sarcoidosis was negative. Diagn. Cytopathol. 2011;39:35-36.

(c) 2010 Wiley-Liss, Inc.

Key Words: asteroid body; cytology; lymph node; granuloma

Asteroid bodies are star-shaped structures found within the cytoplasm of multinucleated giant cells which were originally described in 1911 by Dr. S. B. Wolbach in his article entitled "A New Type of Cell Inclusion, not Parasitic, Associated with Disseminated Granulomatous Lesions" (Fig. C-1). At that time, he described these inclusions in five cases, one from a contributing pathologist from Boston City Hospital, and the remaining four

Department of Pathology, University of Michigan, Ann Arbor, Michigan

*Correspondence to: Stewart M. Knoepp, M.D., Ph.D., Department of Pathology, University of Michigan, 2G332UH, 1500 East Medical Center Dr, SPC 5054, Ann Arbor, MI 48109. E-mail: sknoepp@umich.edu

Received 19 February 2009; Accepted 13 November 2009

DOI $10.1002 /$ dc. 21301

Published online 4 January 2010 in Wiley Online Library (wileyonlinelibrary.com). from a series of 900 autopsies at his Montreal General Hospital. Below is an excerpt of his original description of the new cell inclusion:

"... the type of structure is that of a single mass with radiating straight or curved spinous projections. The central material and the radiating spines have different staining reactions. The central body may be either round or oval in cross section. It is not present in all of the inclusions. The size varies from five or six microns in the smaller inclusions, which consist of a few radially arranged spines, to twenty-five microns in the largest forms, consisting of a central body and radiating straight or curved spines. The number of spines is not constant. The size of the central body, when present, is 2.5 to three micons in diameter. In frozen sections, the whole structure is colorless. They are found most often in giant cells ..."

Although more thorough, his description is similar to what may be seen in textbooks today. Additionally, the questions that he asked nearly a century ago are still of greatest focus today, namely, what are these inclusions and what do they signify? ${ }^{1}$

Wolbach attempted to better characterize these inclusions, now called asteroid bodies, by examining his cases with different processing techniques and the stains available in his day (fat, iron, elastin, and gram stains). Since then many studies have also been designed to answer this question, although the answer is still not completely clear. Ultrastructurally, they are composed of radiating filamentous arms enveloped by a myelin membrane-type material. $^{2}$ Further studies using elemental analysis have revealed the presence of calcium, phosphorous, silicon, and aluminum. ${ }^{3}$

Among his original five cases, Wolbach could find no unifying characteristics to explain the occurrence of these inclusions. They were found in the lymph nodes, spleen, liver, and lungs, and identified in both sexes with age at death ranging from 39 to 72 . Their causes of death were 


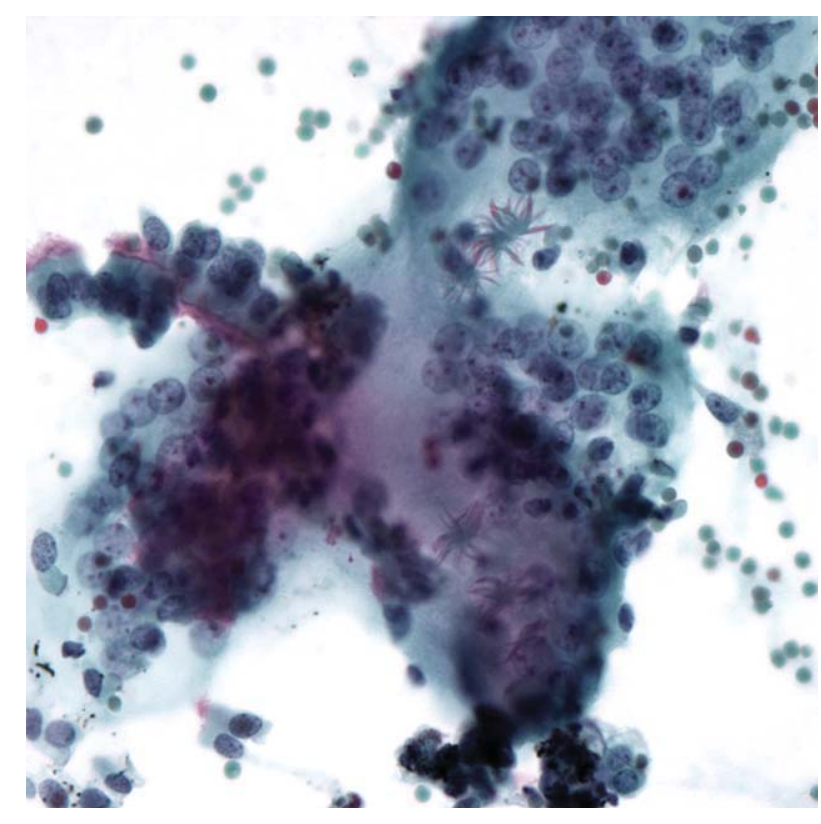

Fig. C-1. Numerous asteroid bodies are seen within the cytoplasm of giant cells from a paratrachial lymph node aspirate. Blood and benign bronchial epithelial cells are present in the background.

just as varied and included carcinomas of the colon and jaw, pernicious anemia, cerebral hemorrhage, and death in the post-operative period following thyroidectomy. Three of the cases had evidence of both cardiac hypertrophy and chronic nephritis and three cases also shared the finding of "chronic pleuritis."

Wolbach excluded mycobacterial infection as the etiology of these inclusions by staining for "tubercle bacilli; always with negative results." He also excluded parasitic infection on the basis that the inclusions were not accompanied by the "crystalline materials" as he had identified in other tissues in cases of parasitic infection. It would be much later that asteroid bodies would be noted to be seen in frequent association with sarcoidosis, ${ }^{4}$ a multisystem granulomatous disease with distinctive clinopathologic features but still of unknown etiology. Additional inclusions associated with sarcoidosis but not seen in the present case are Shaumann bodies (round, concentrically laminated, iron- and calcium-containing inclusions) and Hama-
zaki-Wesenberg bodies (clear, diamond-shaped calcium oxalate crystals and/or ovoid, PAS-positive inclusions). ${ }^{3}$

Wolbach concluded that these inclusions, now called asteroid bodies, were likely the result of an undescribed generalized and perhaps nonspecific process. More than a century later, his speculation has held true; asteroid bodies, as well as the other inclusions mentioned earlier, are quite nonspecific. They have been described in a large variety of granulomatous processes, both caseating and noncaseating and numerous infectious agents and reactive processes have been implicated. ${ }^{3}$

In our case, the most important finding in the lymph node aspirate was that there was no evidence of metastatic carcinoma. This along with the negative workup for sarcoidosis leads to disseminated histoplasmosis as the likely cause of the granulomatous inflammation and asteroid bodies. Histoplasmosis may manifest as granulomatous inflammation long after the organism is eradicated, so granulomas in this setting are not indicative of active disease.

Asteroid bodies are an infrequent finding in fine-needle aspirations (FNAs) ${ }^{5}$ and although nonspecific should raise the concern for sarcoidosis, infection, or malignancy. In Wolbach's original descriptive article, he used the adjectives interesting and peculiar to describe these inclusions and despite not being able to identify their composition or significance, many of his early observations describing them were quite accurate. The case we present is an impressive example of these inclusions now known as asteroid bodies.

\section{References}

1. Wolbach SB. A new type of cell inclusion, not parasitic, associated with disseminated granulomatous lesions. J Med Res 1911;24:243257.

2. Papadimitriou JC, Drachenberg CB. Ultrastructural analysis of asteroid bodies: Evidence for membrane lipid bilayer nature of components. Ultrastruct Pathol 1992;16:413-421.

3. Rosai J. Lymph nodes. In: Rosai and Ackerman's surgical pathology. 9th ed. Mosby; 2004. Vol. 2, Chapter 21, p 1895-1897.

4. Friedman M. Sarcoidosis of the spleen: Report of a case with autopsy and a study of the intracellular "asteroid bodies." Am J Pathol 1944; 20:621-635.

5. Perez-Guillermo M, Sola Perez J, Espinosa Parra FJ. Asteroid bodies and calcium oxalate crystals: Two infrequent findings in fine-needle aspirates of parotid sarcoidosis. Diagn Cytopathol 1992;8:248-252. 